\title{
Performance of genomic medicine
}

\author{
Konrad J Karczewski ${ }^{1,2^{*}}$ and Stephen B Montgomery ${ }^{2,3^{*}}$
}

\section{Abstract \\ A report on the Cold Spring Harbor Laboratory meeting on Precision Medicine: Personal Genomes and Pharmacogenomics, held in Cold Spring Harbor, New York, USA, November 13-16, 2013.}

The third Cold Spring Harbor meeting devoted to clinical applications of genomics covered a broad range of topics from clinical integration and interpretation of genomic variation to advances in genome technology. Unlike previous meetings that focused more on the promise of genomic medicine from its application in a few individuals, speakers highlighted outcomes from developing and deploying more systematic approaches to genomic medicine. The discussed approaches were applied to a variety of clinical applications and cohorts, from interpreting Mendelian disorders to designing individual therapeutic strategies, and from single individuals to 100,000 individuals. In summarizing this meeting, we decided to focus on those talks that provided definitive insight into the performance of genomic medicine, providing a snapshot of where the field is today.

\section{The current state of genomic medicine}

This year truly marked the beginning of full-scale integration of genomics into routine medical practice at a number of institutions. Now that some basic frameworks are in place for generating and returning results, the next step in genomic medicine lies in evaluating how well these models are working. Leslie Biesecker (National Human Genome Research Institute, USA) discussed his success rates from two perspectives. First, in a phenotype-centric view, adopting very high thresholds for variants for cardiomyopathy, he was able to achieve a $100 \%$ positive predictive value, suggesting that the

\footnotetext{
* Correspondence: konradjkarczewski@gmail.com; smontgom@stanford.edu ${ }^{1}$ Biomedical Informatics Training Program, Stanford University School of Medicine, Stanford, CA 94305, USA

${ }^{3}$ Department of Pathology, Stanford University School of Medicine, Stanford, CA 94305, USA

Full list of author information is available at the end of the article
}

pathogenicity criteria are too stringent, resulting in high specificity, but low sensitivity. Alternatively, from a genome-centric view, in 870 exomes primarily unselected for disease, he found 32 variants that were predicted to produce a phenotype. In many cases, after extensive follow-up, patients were found to have phenotypes similar to the prediction, and Biesecker surmised that it was likely that close to 5 to $10 \%$ of people have an undiagnosed Mendelian trait. He went on to discuss an incidental finding in a BRCA1 mutation in a man obtaining a clinical genome for heart disease: on the basis of this information, genetic testing was recommended to the man's sister, who later underwent a laparoscopy to detect fallopian cancer and, after treatment, is now disease free. He urged the audience to 'not be afraid of genomics and preventative medicine' and claimed that genomics is already saving lives.

At this meeting, multiple institutions offered up their overall success rate for diagnosing Mendelian disorders, all of which were in the 25 to $50 \%$ range. As Debbie Nickerson (University of Washington, USA) and others noted, this rate depends on a number of factors, including mode of inheritance, the number of samples available, heterogeneity and knowledge of the phenotype. However, while the ideal success rate would be $100 \%$, she noted that even in mouse models, only approximately $60 \%$ of cases are solved, and so the statistics are better than one might expect. Additionally, indels and large structural variants are crucial in disease and often missed: as methods for detecting these improve, so should the success rate. Liz Worthey (Medical College of Wisconsin, USA) presented her group's initial successful diagnosis rate of approximately $22 \%$, but after reanalysis 18 months later, this number increased to $39 \%$ and even $65 \%$ when including variants of unknown significance. She noted that these numbers are probably underestimates, as the initial cases sent for genome sequencing were already those that were notoriously difficult to solve.

Matthew Hurles (Wellcome Trust Sanger Institute, UK), speaking for the Deciphering Developmental Disorders project in the UK, mirrored these sentiments, and 
provided a detailed breakdown of diagnostic yield for de novo variants among candidate genes for developmental disorders, which, in addition to single nucleotide variants, also included copy number variants, some of which would not have been previously detected by a clinical microarray. Additionally interesting at this meeting was the increasing support for data sharing to enable disease gene discovery. While the issue of genomic data release is a delicate one, Hurles presented the DECIPHER (Database of Chromosomal Imbalance and Phenotype in Humans using Ensembl Resources) consortium's experiences with meeting these challenges to enable sharing of genotypes and phenotypes across the globe. Tim Hubbard (King's College London, UK, and Genomics England, UK) explained a different approach to be used for the $100 \mathrm{~K}$ Genome Project, in which data will be stored behind a firewall at the National Health Service (NHS), but researchers can submit a virtual machine to perform their analyses on the dataset. Nickerson also described their efforts to enable data sharing and patient recruitment to enlarge the sample sizes needed for discovery efforts as part of the Centers for Mendelian Genomics.

From the pharmacogenomics angle, Catherine Brownstein (Boston Children's Hospital, USA) presented their pilot program for pharmacogenetic screening, starting with thiopurine methyltransferase and thiopurines, and integration into the electronic medical record (EMR). She estimated these efforts have already saved many patients from overdosing and saved US $\$ 71,000$ for the hospital for this one drug-gene pair, but also noted there is work to do yet: their current EMRs will not handle the sheer volume of data from a VCF file, and so new challenges are emerging to store these genomic data in the clinic. Brownstein noted that in their clinic, the practitioners have already seen an average of about 2.6 pharmacogenomic clinical decision support alerts. Mary Relling (St Jude Children's Research Hospital, USA) described the PG4KDS pilot program at St Jude Children's Research Hospital, which includes testing for four genes, with results directly integrated into the EMR. She provided an encouraging view on the adoption of these tests, as the program is at nearly $100 \%$ patient compliance, and there does not seem to be evidence of physician alert-fatigue.

\section{The future of genomic medicine}

Throughout the meeting it was evident that there remain multiple challenges to more routine integration of genomics in a clinical setting. While speakers highlighted outcomes in focused areas of application, it was no surprise that these remained a step away from routinely adding each patient's genome to their EMR. While cost/benefit discussions largely factor into why this remains the case, there was also ample discussion as to the desires of patients and doctors when confronted with genomic data. The session on ethics specifically discussed perspectives on the return of secondary results. Robert Green (Brigham and Women's Hospital, USA) shared findings from the REVEAL (Risk Evaluation and Education for Alzheimer's Disease) study that individuals who learn their genetic susceptibilities do not exhibit clinically significant distress. Furthermore, those that learned that they carry risk alleles for two diseases, Alzheimer's and cardiovascular disease, were less worried than individuals who learned they had risk alleles for just one. Concerns about whether this information was causing excessive new challenges for primary care providers (PCPs) or a new burden of unnecessary supplemental testing remain the subject of future focus where all speakers in this session reported ongoing research studies of PCPs to determine the challenges being faced. Green further indicated that this may not be negligible, as up to $28 \%$ of patients indicated they would discuss their results with their PCP. Overall, the ethics session speakers were positive, noting an increase in use of genetic testing for specific applications and medical conditions, along with greater understanding among patients and doctors of its limitations.

Across all sessions it was maintained that healthcare systems and genetic researchers will be facing an everincreasing deluge of genomic data. Organizing and interpreting this flood of data will further require diverse partnerships that span government, academia and industry. In this regard, Tim Hubbard discussed the efforts of Genomics England and the NHS to initiate competition among genomic service providers for the sequencing and interpretation of 100,000 human whole genomes. The benefits of the proposed activity will provide a common, competitive infrastructure for analyzing genomes and health data en masse. Such volumes of genome sequencing data will not be alone, as the Saudi Arabia national science agency has recently proposed to sequence the same number of individuals. In fact, Richard Durbin (Wellcome Trust Sanger Institute, UK) indicated in his plenary talk that the future will require increasing innovation in managing and interpreting large genomics data sets and that by 2020 we can expect to see millions of human genomes sequenced. He further stressed that with these data, we can begin to think about the saturation of genetic information in humans, where all important and non-lethal mutations will be observed in numerous individuals worldwide, a question they are beginning to address with the British Population Autozygosity Gene Function Study. This highlights that methods facilitating the analysis of genomic information across heterogeneous health management settings will be of considerable importance to accelerating the clinical utilization of genomic data in the near future. 


\section{Conclusions}

Future advances in genomic medicine focused on the ongoing innovation in genomic technology. Among the advances presented during the meeting were novel strategies for next generation sequencing variant calling pipelines for clinical applications (Francisco de la Vega, Real Time Genomics, USA), the development of longer read technologies using PacBio sequencing for examination of structural variation (Mark Wang, Baylor College of Medicine, USA) and approaches to incorporate diverse functional genomics data (Ekta Khurana, Yale University, USA). With all of these tools available, the immediate future of genomic medicine will most likely remain focused on tiered sequencing strategies for focused applications.

Abbreviations

EMR: Electronic medical record; NHS: National Health Service; PCP: Primary

care provider.

\section{Competing interests}

The authors declare that they have no competing interests

\section{Author details}

'Biomedical Informatics Training Program, Stanford University School of Medicine, Stanford, CA 94305, USA. ${ }^{2}$ Department of Genetics, Stanford University School of Medicine, Stanford, CA 94305, USA. ${ }^{3}$ Department of Pathology, Stanford University School of Medicine, Stanford, CA 94305, USA.

Published: 23 December 2013

doi:10.1186/gb4146

Cite this article as: Karczewski and Montgomery: Performance of genomic medicine. Genome Biology 2013 14:316. 